\title{
Topology Optimization in Electric Car Body Frame Based on Optistruct
}

\author{
Dongdong $\mathrm{Ge}^{1}$,Liangrong Zhu ${ }^{1}$, Dongji Xuan ${ }^{2}$ \\ ${ }^{1}$ Institute of vehicle, Zhejiang Industry Polytechnic College, Shaoxing 312000, China; \\ ${ }^{2}$ College of Mechanical \& Electrical Engineering, Wenzhou University, Wenzhou 325035, China \\ agdd1988@126.com, b348607826@qq.com, ${ }^{c}$ xuandongii@163.com
}

\begin{abstract}
In order to optimize the structure of the electric car body frame, the static analysis of the car frame were carried on. For the goal of the frame's weight minimum, OptiStruct software was used to topology optimization design. And the optimal material distribution program of the frame structure was got. Static strength before and after optimization was comprehensive compared through the stress, deformation. The results showed that the weight of frame after optimization was reduced by $18.96 \%$,and the requirements of the strength and stiffness were ensured.
\end{abstract}

Keywords. Electric car, static analysis, OptiStruct, topology optimization.

\section{Introduction}

The goal of structure optimation is achieving the optimal performance of the structure, including objectives such as strength, stiffness and stability by using the least amount of material, the most simple process, low cost 1 .Depending on the simulation experiment and the experience of the traditional optimization design method is difficult to solve the design problems such as plane, automobile2, ship, mold3 and other large-scale complex structure parts and bridge4,tunnel and other large engineering. Topology optimization method is becoming one of the key means to solve such problems 5 .

The insufficient mileage is one of the major bottleneck factors which affect the promotion and application of the electric cars. This study based on the electric car body frame static analysis, the minimum body frame weight as the optimization goal,OptiStruct software was used to topology optimization design, get the optimal material distribution program of body frame structure.Finally HyperWorks and general numerical analysis software Radioss were used to static analysis of body frame before and after optimization, from the stress, deformation, weight and other aspects of the calculation results were compared.

\section{Static analysis of the electric car body frame}

\subsection{The analysis model}

In this paper, UG software was used to model one electric car (shown in figure 1), and body frame model (shown in figure 2) was isolated.The total mass of lower body frame part is $17.88 \mathrm{Kg}$, a total of 17 beams. Based on the finite element technology, using Hypermesh software to carry on finite element modeling of the body frame. Radioss software was used to make static analysis on the body frame under four kinds of typical working conditions. (horizontal bending condition, emergency braking condition, ultimate torsion condition, emergency turn condition)

The material of electric car body frame is Q345. Youngs's modulus, Poisson's ratio,density,thickness for the material of body frame is taken as $210 \mathrm{Gpa}, 0.3,7800 \mathrm{~kg} / \mathrm{m}^{3}$ and $2 \mathrm{~mm}$. 


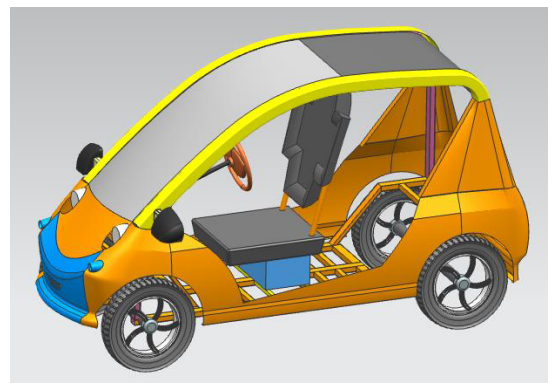

Fig. 1. Electric car body model of UG

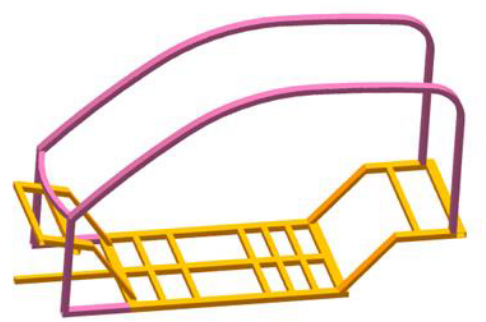

Fig. 2. Body frame model

\subsection{Analysis of typical working condition}

The electric car body frame material is Q345, and failure marks is plastic deformation.Make analysis of static strength and stiffness according to Von-Misses stress distribution and displacement deformation of the body frame. Referring to the literature6, 7 and 8 on the treatment of boundary conditions, make static analysis on the body frame under four kinds of typical working conditions. By analyzing the stress and displacement response of the body frame conditions, improve unreasonable structure, and provide the basis for lightweight design body frame structure.

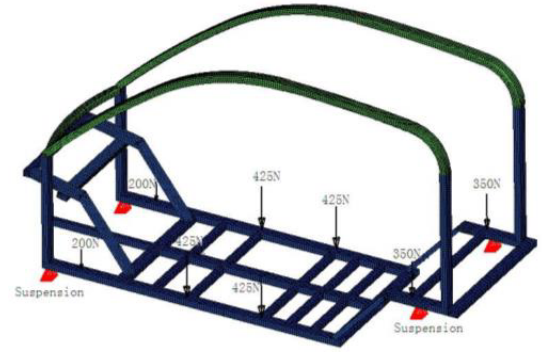

Fig. 3. The boundary conditions of electric car body frame

According the car model suspension, seat, power, occupant layout (Shown in figure 3), set the boundary conditions of the body frame. The total weight of driver and battery are $1700 \mathrm{~N}$, vertical load in the central body frame with 4 average concentration $(425 \mathrm{~N})$. Weight of rear passenger is $700 \mathrm{~N}$, vertical load in the rear body frame with 2 average concentration $(350 \mathrm{~N})$. Weight of front mechanism is $400 \mathrm{~N}$, vertical load in the front body frame with 2 average concentration (200N). The freedom restricted position of front and rear suspension is the car suspension lug actual installation location.

(1)Horizontal bending condition 678

Setting up forces on the body frame under the horizontal bending condition, DOF constraints suspension system.Constraint nodes at the left front suspension UX, UY, UZ freedom; Constraint nodes at the right front suspension UY, UZ freedom;Constraint nodes at the left rear suspension UX,UZ freedom; Constraint nodes at the right rear suspension UZ freedom, and release of other degrees of freedom.

(2)Emergency braking condition 67

The vertical load of this condition is the same as horizontal bending condition.Apply a $0.8 \mathrm{~g}$ brake acceleration in the longitudinal direction of the model.Constraint nodes at the front suspension UX, UY, UZ freedom;Constraint nodes at the rear suspension UY, UZ freedom, and release of other degrees of freedom.

(3)Ultimate torsion condition 678 
This condition is mainly to simulate the car round dangling or round up. Left and right wheels have height difference. The car body is subjected to greater torsion under this condition, and it is one of the most serious conditions of the car.

Left front wheel round dangling to simulate this working condition. Constraint nodes at the right front suspension UX, UY, UZ freedom; Constraint nodes at the rear suspension UZ freedom; Release all degrees of freedom at the left front suspension.

Figure 4 and 5 are the stress diagram, deformation displacement diagram under ultimate torsion condition.

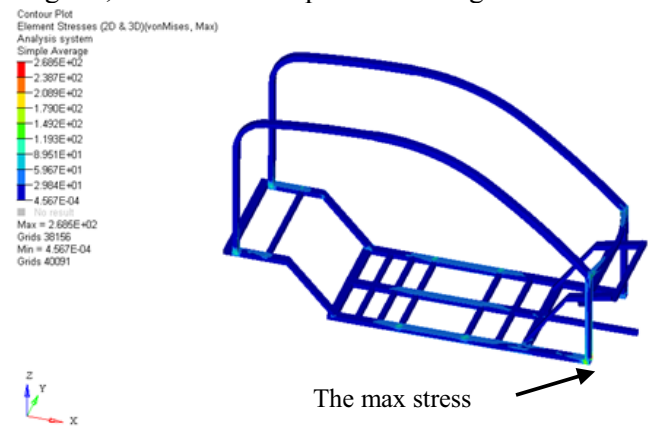

Fig. 4. Stress diagram of ultimate torsion condition

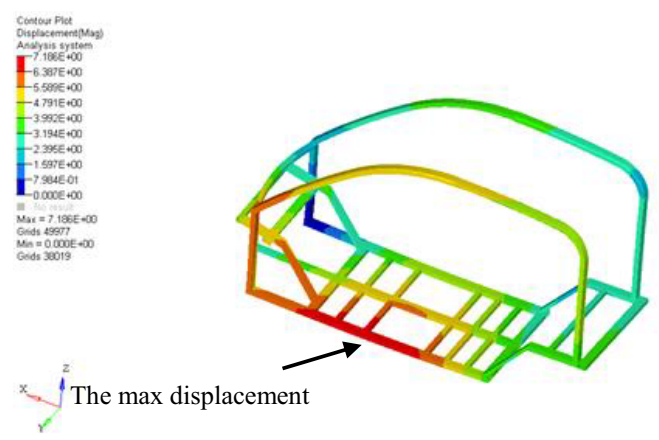

Fig. 5. Deformation of ultimate torsion condition

(4)Emergency turn condition 67

This condition is mainly to simulate the responsiveness of body frame at the lateral inertial force and vertical load when the car emergency turns.Apply a $0.4 \mathrm{~g}$ lateral acceleration which simulates lateral load in the centroid of body frame.

Constraint nodes at the left front suspension UX,UY, UZ freedom; Constraint nodes at the right front suspension UY, UZ freedom; Constraint nodes at the rear suspension UY, UZ freedom, and release of other degrees of freedom.

Table 1 shows maximum stress and maximum deformation of the body frame in 4 typical working conditions.

Table 1. Comprehensive analysis of 4 typical working conditions

\begin{tabular}{|c|c|c|}
\hline Condition & Max stress(MPa) & $\begin{array}{c}\text { Max deformation } \\
\text { (mm) }\end{array}$ \\
\hline Horizontal bending & 144 & 3.7 \\
\hline Emergency braking & 268 & 7.2 \\
\hline Ultimate torsion & 275 & 9.4 \\
\hline Emergency turn & 147 & 4.5 \\
\hline
\end{tabular}

\section{Topology optimization in body frame}

Based on the electric car body frame static analysis, the minimum body frame weight as the optimization goal, OptiStruct software was used to topology optimization design, get the optimal material distribution program of body frame structure. Finally HyperWorks and Radioss were used to static analysis of body frame before and after optimization. 


\subsection{Topology optimization}

Figure 6 is topology optimization design process of an electric car body frame 8 .

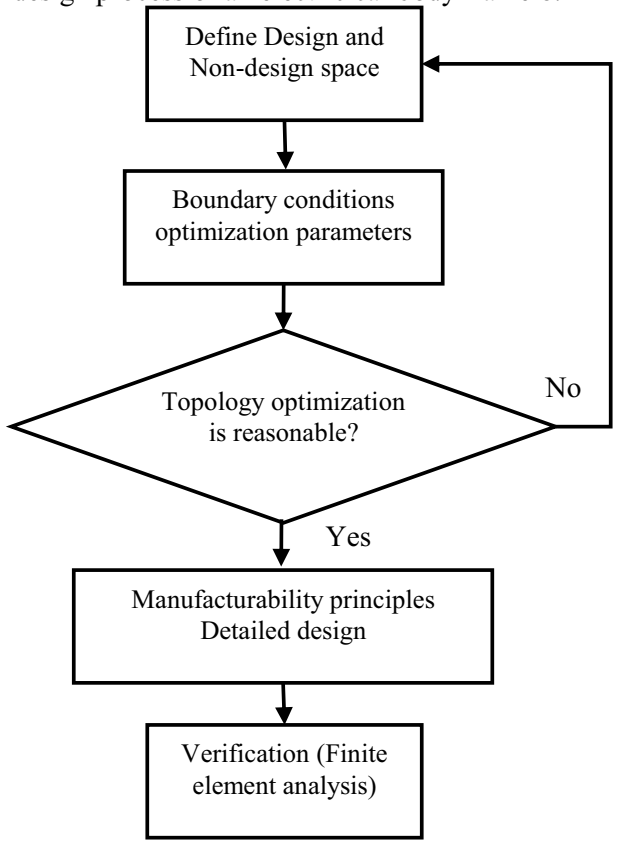

Fig. 6. Design process of topology optimization in body frame

Based on design process of topology optimization to define the design space and non-design space.Non-design space is some have been identified in the structure, which will not be changed in the design process.In Figure 7 (a) is non-design space. Figure 7 (b) is design space(part of the blue region in the model).

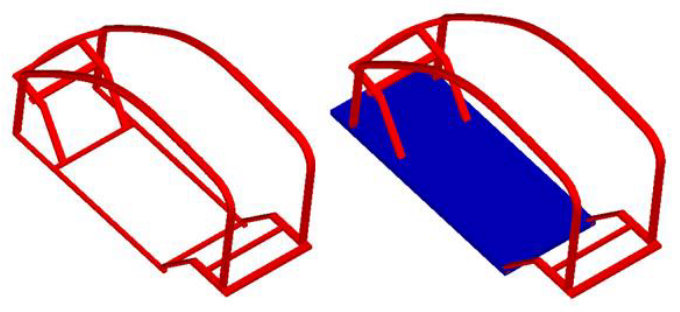

(a) Non-design space (b) Design space(Part of the blue area)

Fig. 7. The non-design space and design space of body frame

The car body is subjected to greater torsion under ultimate torsion condition, and it is one of the most serious conditions of the car.To meet the stiffness requirements of ultimate torsion conditions, we set the boundary conditions, optimization parameters and submit calculation, then get the topology optimization program.

Accordance with the previous ultimate torsion condition set boundary conditions.Optimization parameters include design variables, response function, design objective and design constraints.Element pseudo-density is design variables, the total mass and nodes(Position of arrow in Fig. 5) in the Z-direction displacement are the response functions.Minimum total mass of the model is design objective. The node maximum allowable displacement $7.2 \mathrm{~mm}$ is design constraint.By constraining displacement of the body frame to ensure rigidity of the body frame.Optimization mathematical model is as follows.

$$
\left\{\begin{array}{l}
\operatorname{Min} W\left(\beta_{i}\right)=\sum_{i} \beta_{i} \rho_{i} v_{i} \\
\text { s.t. }\left\{\begin{array}{l}
d_{j} \leq 7.2 \\
\beta_{i} \in[0,1]
\end{array}\right.
\end{array}\right.
$$


$W$ is the total mass of the model; $\beta_{i}$ is design variable; $\rho_{i}$ is element initial density of number $i ; v_{i}$ is element volume of number $i ; d_{j}$ is nodes in the Z-direction displacement.

This optimization process takes 23 iterations,and fig. 8 shows topology result.After analysising this topology result, some nodes maximum deformation displacement of body frame reach $16 \mathrm{~mm}$. So this topology optimization program has failed to fully meet the requirements of the initial stiffness of the body frame.

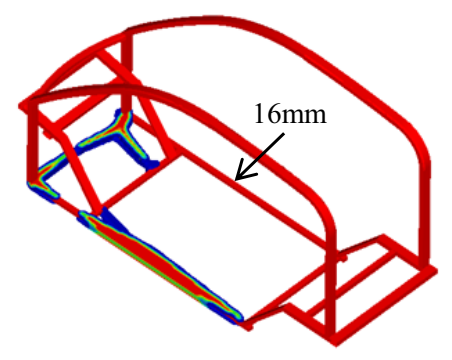

Fig. 8. Topology optimization of single-node constraint in body frame

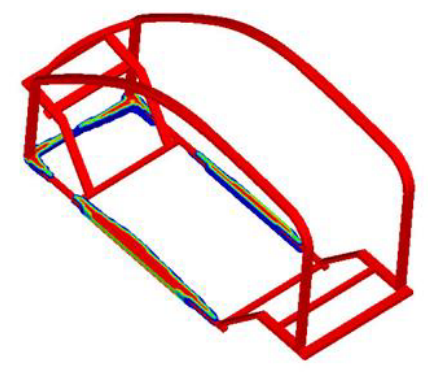

Fig. 9. Topology optimization of multi-nodes constraint in body frame

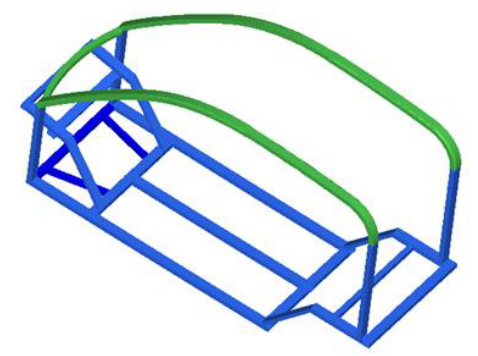

Fig. 10. Detailed design of body frame

According to the first results of the topology optimization, adjust optimization parameters. Element pseudo-density is design variables, the total mass and nodes(Positions of arrow in Fig.5 and Fig.8) in the Z-direction displacement are the response functions.Minimum total mass of the model is design objective.The nodes maximum allowable displacement 7.2 $\mathrm{mm}$ is design constraint.

The second optimization process takes 23 iterations,and fig.9 shows topology result.After analysising this topology result, all nodes maximum deformation displacement of body frame less than $7.2 \mathrm{~mm}$. Topology optimization program meet the requirements of the initial stiffness of the body frame.

Based on two times topology optimization results about material distribution of body frame, and according to the principle of manufacturability detailed design the body frame.Figure 10 shows detailed design of body frame. The total mass of the lower part of the body frame is $14.49 \mathrm{Kg}$, a total of 8 beams after optimization.

\subsection{Finite Element Verification and contrast}


HyperWorks and Radioss were used to static analysis of body frame before and after optimization under four kinds of typical working conditions(horizontal bending condition, emergency braking condition, ultimate torsion condition, emergency turn condition), from the stress, deformation, weight and other aspects of the calculation results were compared.

Table 2. Comparison of static strength stiffness before and after optimization

\begin{tabular}{|c|c|c|c|}
\hline \multirow{2}{*}{$\begin{array}{c}\text { Condition } \\
\text { Horizontal } \\
\text { bending }\end{array}$} & Before & $\begin{array}{c}\text { Max stress } \\
\text { (MPa) }\end{array}$ & $\begin{array}{c}\text { Max } \\
\text { deformation } \\
\text { ( mm) }\end{array}$ \\
\cline { 2 - 4 } & After & 144 & 3.7 \\
\hline \multirow{2}{*}{$\begin{array}{c}\text { Emergency } \\
\text { braking }\end{array}$} & Before & 268 & 3.7 \\
\cline { 2 - 4 } & After & 279 & 7.2 \\
\hline \multirow{2}{*}{$\begin{array}{c}\text { Ultimate } \\
\text { torsion }\end{array}$} & Before & 275 & 9.4 \\
\cline { 2 - 4 } & After & 286 & 9.0 \\
\hline \multirow{2}{*}{ Emergency turn } & Before & 147 & 4.5 \\
\cline { 2 - 4 } & After & 144 & 4.6 \\
\hline
\end{tabular}

The results showed that after optimizion the body frame meet the strength and stiffness requirements under four kinds of typical working conditions. The total mass of lower part of the body frame reduced from $17.88 \mathrm{KG}$ to $14.49 \mathrm{KG}$,reduced $18.96 \%$; The number of beams reduced from 17 to 8 , and the length of the weld was ignificantly reduced.

Literature 8, 9 and 10 verify the effectiveness of above design process of topology optimization in body frame. The way which apply Optistruct software on the body frame to topology optimization design is an effective way to solve the problem of automobile lightweight.

\section{Summary}

This paper chooses the electric car body frame as the research object, using Radioss software on the body skeleton four typical conditions of static analysis. Radioss software was used to make static analysis on the body frame under four kinds of typical working conditions.OptiStruct software was used to topology optimization design, get the optimal material distribution program of body frame structure.Finally HyperWorks and Radioss were used to static analysis of body frame before and after optimization, from the stress, deformation, weight and other aspects of the calculation results were compared.The results showed that after optimizion the body frame meet the strength and stiffness requirements under four kinds of typical working conditions. The total mass of lower part of the body frame reduced from $17.88 \mathrm{KG}$ to $14.49 \mathrm{KG}$, reduced $18.96 \%$; The number of beams reduced from 17 to 8 ,and the length of the weld was ignificantly reduced.

\section{References}

1. H.S. Ho, B. Lui, X.H. Xing, Parametric Shape and Topology Optimization with Radial Basis Functions and Partition of Unity Method.American Institute of Physics, (2010)

2. H.S. Lee, A structural analysis and topology optimization on cylinder block of heavy duty diesel engine.International Journal of Modern Physic B., 24, 15(2010)

3. Nilsson, F. Birath, Topology Optimization Of A Stamping Die.Materials Processing and Design, (2007)

4. F.Gerold, K.Beucke, F.Seible, M. Asce, Integrative Structural Design.Journal of Computing in Civil Engineering, 26, 6(2012)

5. S. Amstutz, Analysis of a level set method for topology optimization.Optimization Methods \& Software, 26, 4(2011)

6. Y.B. Zhang, Full load bus body design analysis and research.Zhejiang University,(2008)

7. X.N. Zuo, Analysis and lightweight research of coach body frame.Harbin Institute of Technology,(2011) 
8. X.Y.Xu, Y.J.Guo, Stiffness constrained topology optimization for body structure of electric low speed car. Machine Design and research, 26, 2(2010)

9. W.J. Fan, Z.J. Fan, R.Y. Su, Research on Multi-objective Topology Optimization on Bus chassis frame.Journal of mechanical engineering, 19, 12(2008).

10. W.J. Fan, Z.J. Fan, Muti-stiffness topology optimization of bus frame with multiple loading conditions.Automotive Engineering, 30, 6(2008) 